\title{
Chronic but not intermittent infection with Pseudomonas aeruginosa is associated with global changes of the lung microbiome in cystic fibrosis
}

To the Editor:

Chronic pulmonary infection with Pseudomonas aeruginosa that triggers chronic airway inflammation and structural lung damage remains a key risk factor for morbidity and mortality in patients with cystic fibrosis (CF) [1]. Previously, culture-based diagnostics considered airway infections in CF patients as mono-pathogenic, but recent approaches using culture-independent methods showed that bacterial infection of the CF lung involves a polymicrobial community [2-4]. Chronic infection with typical CF pathogens like $P$. aeruginosa is associated with a decline in lung function and correlates with a decrease of diversity in the lung microbiota. However, the interactions between pathogens and the microbiota remains poorly understood, and it has been hypothesised that dysbiosis of the microbiota may pre-exist and facilitate growth of pathogens such as $P$. aeruginosa in the CF lung. The aim of the current study was to evaluate the influence of $P$. aeruginosa infection on the dysbiosis of the microbiota in the CF lung.

This study was approved by the ethics committee of the University of Heidelberg and informed written consent was obtained from the study subjects. Airway samples from patients with CF were obtained during quarterly routine outpatient visits $(n=336)$ and during hospitalisation $(n=56)$. The diagnosis of CF was based on established criteria [5], and infection with P. aeruginosa was classified as negative, intermittent or chronic, as previously described [6]. In total, 392 sputum samples from 71 patients with CF with a broad age range (mean \pm SD age $21 \pm 11$ years, range 3-70 years) and disease severity (mean \pm SD forced expiratory volume in $1 \mathrm{~s}$ (FEV1) predicted $55.7 \pm 24.9 \%$, range 13.1-131.9\%), including longitudinal samples from 50 subjects, were analysed at various stages of $P$. aeruginosa infection; 154 negative, 88 intermittent and 150 chronic infection samples were included. Moreover, 408 throat swabs were analysed from $71 \mathrm{CF}$ patients (268 negative, 77 intermittent and 63 chronic infections). DNA was extracted, amplified using 16S rRNA V4 region, sequenced and computationally and statistically analysed as previously described [7].

Sputum samples from patients with chronic P. aeruginosa infection (Psa chronic) were distinct from those from the group without infection (Psa negative) and the group with intermittent infection (Psa intermittent) in principal coordinates analysis (figure 1a and b). PERMANOVA showed that only the Psa chronic group was significantly different from the two other groups $(\mathrm{p}<0.01)$. Moreover, Psa chronic sputum samples showed a significantly lower diversity $(\mathrm{p}<0.01)$ (figure $1 \mathrm{c}$ ) and a higher dominance $(\mathrm{p}<0.01)$ than the Psa negative or Psa intermittent sputa, and an increase in total biomass of bacteria $(\mathrm{p}<0.05)$ when compared to the Psa negative group. Hierarchical clustering indicated that 12 clusters most effectively modelled the population (figure 1a). Most of the Psa chronic samples $(80.0 \%)$ were found in one cluster which was characterised by a high abundance of $P$. aeruginosa (cluster Psae). Two other major clusters were identified, either characterised by a high abundance of Neisseria, Veillonella and Prevotella (cluster NVP), known commensals of the oropharynx, or dominated by Staphylococcus (cluster Sta). Additional minor clusters were characterised by the dominance of other classical respiratory pathogens including Stenotrophomonas (Ste), Burkholderia (B2), Mycoplasma (M), Bordetella (B1) or commensals from the upper airways (Prevotella (P), Rothia (R)). In all, 54.5\% of the Psa negative and 51.1\% of the Psa intermittent samples clustered in the NVP cluster (figure 1d). A multivariate analysis (including age, gender and exacerbation status) of the three major

@ERSpublications

Chronic but not intermittent $P$. aeruginosa infection results in dysbiosis of lung microbiota in cystic fibrosis http://ow.ly/cDKO30eOnx5

Cite this article as: Boutin S, Graeber SY, Stahl M, et al. Chronic but not intermittent infection with Pseudomonas aeruginosa is associated with global changes of the lung microbiome in cystic fibrosis. Eur Respir J 2017; 50: 1701086 [https://doi.org/10.1183/13993003.01086-2017]. 


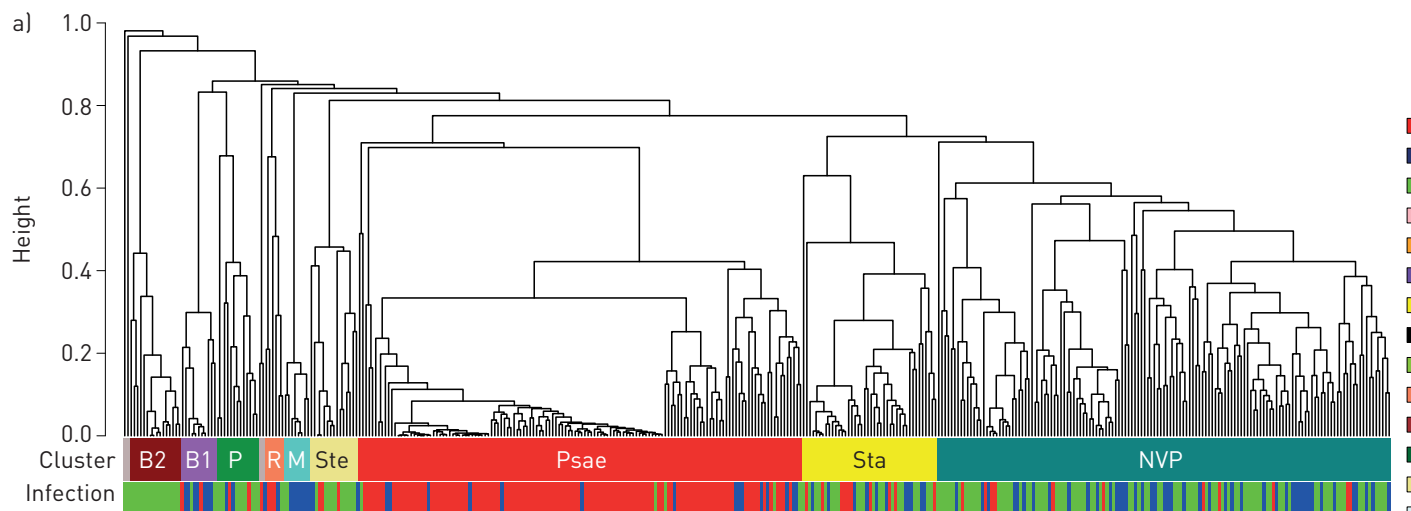

$\square$ Otu00001 (Pseudomonas)

- Otu00002 (Prevotella)

Otu00003 (Streptococcus)

$\square$ Otu00004 (Haemophilus)

$\square$ Otu00005 (Neisseria)

$\square$ Otu00006 (Veillonella)

$\square$ Otu00007 (Staphylococcus)

- Otu00008 (Prevotella)

$\square$ Otu00009 (Fusobacterium)

$\square$ Otu00010 (Rothia)

$\square$ Otu00011 (Gemella)

Otu00015 (Moraxella)

Infection

$\square$ Otu00016 (Stenotrophomonas)

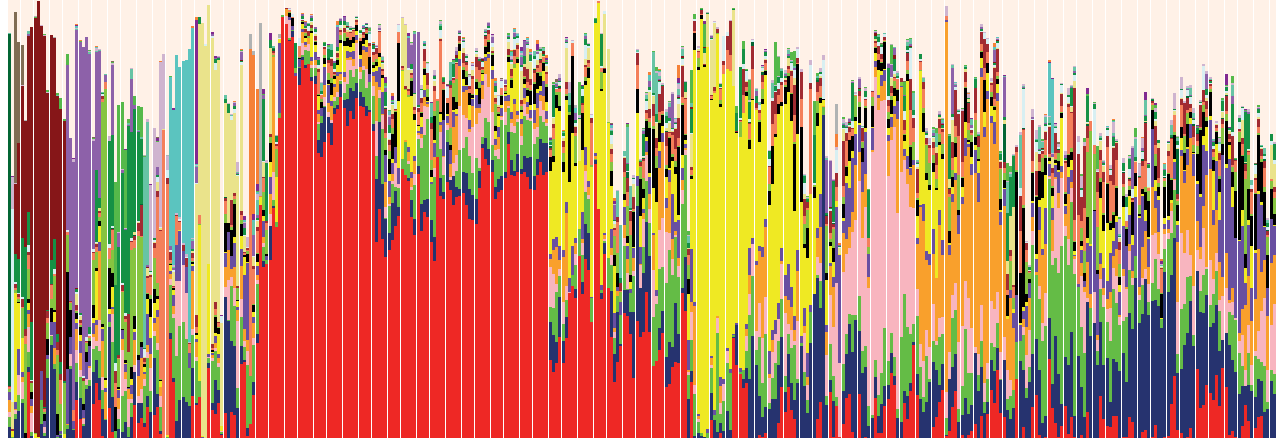

$\square$ Otu00018 (Streptococcus)

$\square$ Otu00019 (Prevotella)

Otu00022 (Burkholderia)

$\square$ Otu00023 (Bordetella)

$\square$ Otu00032 (Capnocytophaga)

$\square$ Otu00033 (Granulicatella)

$\square$ Otu00035 (Mycoplasma)

$\square$ Otu00041 (Prevotella)

Otu00045 (Acinetobacter)

$\square$ Otu00061 (Burkholderia)

$\square$ Otu00062 (Escherichia-Shigella)

$\square$ Otu00101 (Lactobacillus)

$\square$ Other

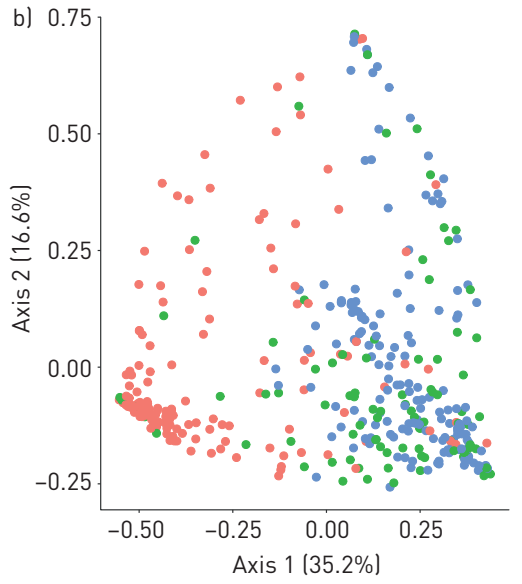

Pseudomonas status

- Chronic Intermittent Negative

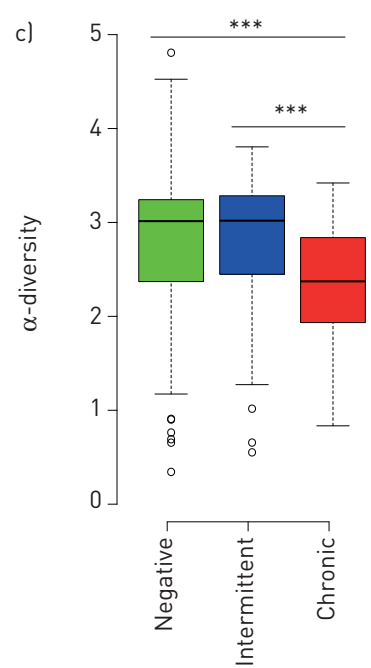

Pseudomonas status
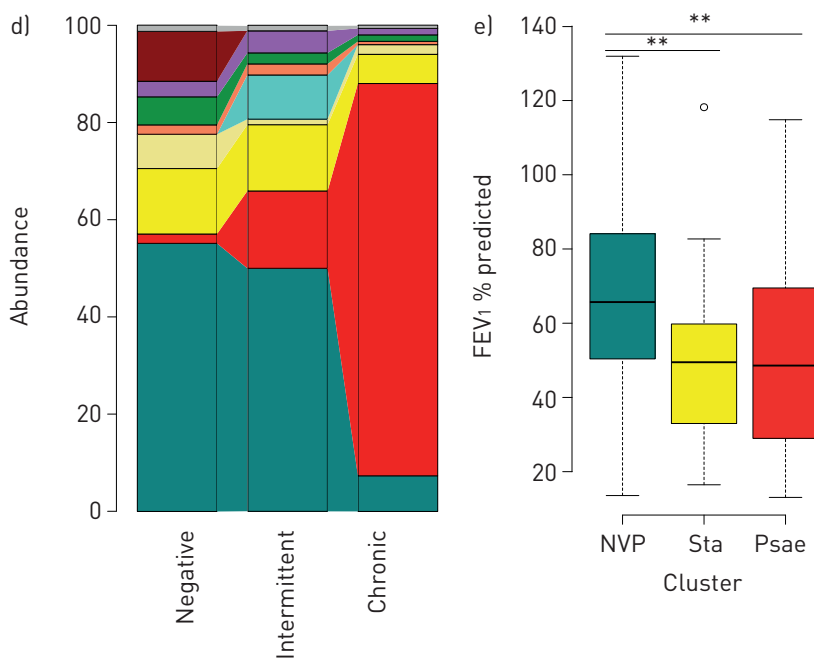

FIGURE 1 Clustering of sputum microbiota in patients with cystic fibrosis. a) Clusters are represented in colour and are based on the Morisita-Horn distance represented by the dendrogram. The bottom is a stack bar plot of the relative abundances of the 25 most abundant $0 T$ T. Clusters are named by the most abundant genus: NVP: Neisseria/Veillonella/Prevotella, Sta: Staphylococcus, Psae: Pseudomonas, Ste:

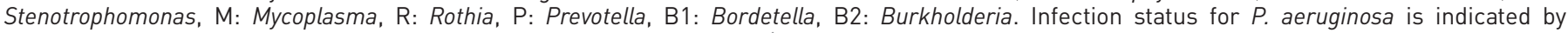
colours: negative in green, intermittent in blue and chronic in red. b) Principal coordinates analysis representing the Morisita-Horn distance between samples from patients without $P$. aeruginosa infection (negative) and patients with intermittent and chronic $P$. aeruginosa infection. $c$ ) $\alpha$-diversity related to infection status for $P$. aeruginosa as assessed by the non-parametric Shannon index. $d)$ Percentage of samples belonging to each cluster per infection status (negative, intermittent and chronic). e) Forced expiratory volume in $1 \mathrm{~s}$ (FEV1) \% predicted of patients belonging to the three major clusters: NVP. Sta and Psae. Statistical analyses were performed by a multivariate analysis lincluding age, gender and exacerbation status). ${ }^{* *}: p<0.01 ;{ }^{* * *}: p<0.001$.

clusters (NVP, Psae and Sta) was performed to compare FEV1 \% pred and body mass index (BMI) standard deviation score between the clusters. In patients with a sputum microbiome clustering in the Pseudomonas and Staphylococcus groups, FEV1 \% pred was significantly reduced $(\mathrm{p}<0.01)$ as compared to patients carrying 
an NVP-dominated microbiome (figure 1e). Furthermore, including all samples with a lung function evaluation (292 samples from 68 patients), a negative correlation of FEV1 \% pred with dominance (which is the relative abundance of the most abundant operational taxonomic unit (OTU) (Berger-Parker index [8]), $\mathrm{R}^{2}=-0.26, \mathrm{p}<0.001$ ) and a positive correlation with alpha-diversity (nonparametric Shannon index which takes into account the number of OTUs and the abundance distribution [9], $\mathrm{R}^{2}=0.33, \mathrm{p}<0.001$ ) could be shown. The BMI was not significantly different between CF patients from the three different clusters. In the throat, no changes in the microbiome were observed in respect to Pseudomonas infection status.

Our study shows that chronic but not intermittent infection with $P$. aeruginosa was associated with dysbiosis in the lungs of patients with CF. Patients with chronic P. aeruginosa infection showed a global decrease in alpha-diversity and a specific decrease of genera including Prevotella, Neisseria and Veillonella. Those genera are the major components of the ecotype NVP (respective linear discriminant analysis (LDA)-score: $3.92,4.65,4.30$ ), a highly diverse microbiota closely related to the throat microbiota observed in this study (data not shown), as well as in healthy people [10]. This result is in line with the current theory stating that the lung microbiome is a result of balancing migration/clearance from the oropharyngeal microbiota [11]. Despite reports arguing for a disease-promoting role of this microbiota in CF, others suggested that those commensals reflect the normal lung microbiome and might even be beneficial by ecological competition with CF pathogens $[3,10,12,13]$. This ecotype is mostly found in patients with absent or intermittent infection with $P$. aeruginosa. Intermittent infection can be interpreted as the first encounter or new re-infection with $P$. aeruginosa which, according to our data, is not accompanied with changes of the underlying microbiome in the CF lung. These findings are consistent with a model in which colonisation with $P$. aeruginosa is the primary driver in the development of an abnormal lung microbiome that is associated with increased disease severity in patients with CF [1, 14]. Patients with intermittent $P$. aeruginosa infection did not exhibit major alterations in the lung microbiota, arguing against the hypothesis that alterations in the microbiome are a precursor for Pseudomonas infection. This sequence of changes highlights that early detection and eradication of $P$. aeruginosa may limit or even prevent progression of profound dysbiosis and thereby, the establishment of a disease-promoting microbiome in the CF lung.

Similar to the alterations observed with $P$. aeruginosa, clusters with dominance of other genera including Staphylococcus, Stenotrophomonas, Burkholderia, Mycoplasma and Bordetella were also associated with decreased diversity and increased dominance of the lung microbiome. Of note, dominance was negatively correlated to lung function as described in previous studies [3, 15]. Furthermore, FEV1 \% pred was significantly lower in patients dominated by Pseudomonas and Staphylococcus infections than those from the NVP cluster. These data suggest that dominance of one species remodels the lung microbiota and may promote severity of CF lung disease, whereas the highly diverse NVP cluster might be protective and could correlate with a better outcome.

A limitation of our study is that $16 \mathrm{~S}$ amplicon sequencing does not allow the identification of mechanisms leading to chronic infection with $P$. aeruginosa, or other pathogens identified in our cluster analysis, and induction of dysbiosis in the CF lung. Future studies analysing metagenomics and transcriptomics will be needed to better understand the underlying mechanisms of these alterations. Our cluster analysis could help to select defined samples for such an approach.

In summary, this study indicates that intermittent $P$. aeruginosa infection occurs without dysbiosis in $\mathrm{CF}$ lung disease, and chronic $P$. aeruginosa infection triggers dysbiosis in the lung. Therefore, we hypothesise that early eradication of $P$. aeruginosa during intermittence may preserve a more diverse and beneficial lung microbiome, thereby contributing to clinical benefits in patients with $\mathrm{CF}$.

Sébastien Boutin ${ }^{1,2}$, Simon Y. Graeber ${ }^{2,3,4}$, Mirjam Stahl ${ }^{2,3,4}$, A. Susanne Dittrich ${ }^{2,4,5}$, Marcus A. Mall ${ }^{2,3,4,6}$ and Alexander H. Dalpke $\oplus^{1,2,6}$

${ }^{1}$ Dept of Infectious Disease, Medical Microbiology and Hygiene, University Hospital Heidelberg, Germany. ${ }^{2}$ Translational Lung Research Center Heidelberg (TLRC), German Center for Lung Research (DZL), University of Heidelberg, Heidelberg, Germany. ${ }^{3}$ Division of Pediatric Pulmonology and Allergy and Cystic Fibrosis Center, Dept of Pediatrics, University of Heidelberg, Heidelberg, Germany. ${ }^{4}$ Dept of Translational Pulmonology, University of Heidelberg, Heidelberg, Germany. ${ }^{5}$ Dept of Pneumology and Critical Care Medicine, Thoraxklinik at the University Hospital Heidelberg, Heidelberg, Germany. ${ }^{6}$ Contributed equally as senior authors.

Correspondence: Alexander H. Dalpke, Dept of Infectious Diseases, Medical Microbiology and Hygiene, Heidelberg University Hospital, Im Neuenheimer Feld 324, 69120 Heidelberg, Germany.

E-mail: alexander.dalpke@med.uni-heidelberg.de

Received: Mar 142017 | Accepted after revision: July 042017

Support statement: This study was supported in part by the German Ministry for Education and Research (82DZL00401, 82DZL004A1) and from the European Commission (Seventh Framework Programme Project No. 
603038, CFMatters). A.S. Dittrich is the recipient of a HRCMM (Heidelberg Research Center for Molecular Medicine) Career Development Fellowship. Funding information for this article has been deposited with the Crossref Funder Registry.

Conflict of interest: Disclosures can be found alongside this article at erj.ersjournals.com

Acknowledgements: We acknowledge the excellent technical support by Jessica Panitz, Selina Hassel, David Ibbersson and the technical team from Bioquant. We are also grateful to Iris Kühbandner for patient recruitment and sample collection.

Author contributions: Conception and design of the study: S. Boutin, S.Y. Graeber, M. Stahl, A.S. Dittrich, M.A. Mall, A.H. Dalpke; acquisition, analysis and interpretation of the data: S. Boutin, S.Y. Graeber, M. Stahl, A.S. Dittrich, M.A. Mall, A.H. Dalpke; drafting the article or revising it critically for important intellectual content: S. Boutin, S.Y. Graeber, M. Stahl, A.S. Dittrich, M.A. Mall, A.H. Dalpke.

\section{References}

$1 \quad$ Mall MA, Hartl D. CFTR: cystic fibrosis and beyond. Eur Respir J 2014; 44: 1042-1054.

2 Tunney MM, Field TR, Moriarty TF, et al. Detection of anaerobic bacteria in high numbers in sputum from patients with cystic fibrosis. Am J Respir Crit Care Med 2008; 177: 995-1001.

3 Boutin S, Graeber SY, Weitnauer M, et al. Comparison of microbiomes from different niches of upper and lower airways in children and adolescents with cystic fibrosis. PLOS One 2015; 10: e0116029.

4 Zhao J, Elborn JS, LiPuma JJ. Airway infection and the microbiome. In: Mall MA, Elborn JS, eds. Cystic Fibrosis (ERS Monograph). Sheffield, European Respiratory Society, 2014; pp. 32-64.

5 De Boeck K, Derichs N, Fajac I, et al. New clinical diagnostic procedures for cystic fibrosis in Europe. J Cyst Fibros 2011; 10: Suppl. 2, S53-S66.

6 Stahl M, Wielpütz MO, Graeber SY, et al. Comparison of lung clearance index and magnetic resonance imaging for assessment of lung disease in children with cystic fibrosis. Am J Respir Crit Care Med 2016; 195: 349-359.

7 Boutin S, Hagenfeld D, Zimmermann $\mathrm{H}$, et al. Clustering of subgingival microbiota reveals microbial disease ecotypes associated with clinical stages of periodontitis in a cross-sectional study. Front Microbiol 2017; 8: 340.

8 Berger WH, Parker FL. Diversity of planktonic foraminifera in deep-sea sediments. Science 1970; 168: 1345-1347.

9 Chao A, Shen T-J. Nonparametric estimation of Shannon's index of diversity when there are unseen species in sample. Environ Ecol Stat 2003; 10: 429-443.

10 Charlson ES, Bittinger K, Haas AR, et al. Topographical continuity of bacterial populations in the healthy human respiratory tract. Am J Respir Crit Care Med 2011; 184: 957-963.

11 Dickson RP, Erb-Downward JR, Freeman CM, et al. Spatial variation in the healthy human lung microbiome and the adapted island model of lung biogeography. Ann Am Thorac Soc 2015; 12: 821-830.

12 Sherrard LJ, Bell SC, Tunney MM. The role of anaerobic bacteria in the cystic fibrosis airway. Curr Opin Pulm Med 2016; 22: 637-643.

13 O’Neill K, Bradley JM, Johnston E, et al. Reduced bacterial colony count of anaerobic bacteria is associated with a worsening in lung clearance index and inflammation in cystic fibrosis. PLOS One 2015; 10: e0126980.

14 Adler FR, Liou TG. The dynamics of disease progression in cystic fibrosis. Plos One 2016; 11: e0156752.

15 Cox MJ, Allgaier M, Taylor B, et al. Airway microbiota and pathogen abundance in age-stratified cystic fibrosis patients. PloS One 2010; 5: e11044. 